\title{
Development of Attentional Functions in School-Age: Evidence from both Traditional and Computerized Tasks
}

\author{
Amaryllis-Chryssi Malegiannaki ${ }^{1} \&$ Panayiota Metallidou ${ }^{1}$ \\ ${ }^{1}$ School of Psychology, Aristotle University of Thessaloniki, Thessaloniki, Greece \\ Correspondence: Amaryllis-Chryssi Malegiannaki, School of Psychology, Aristotle University of Thessaloniki, \\ Thessaloniki, 54124, Greece. Tel: 30-231-099-7972. E-mail: malegiannaki_amaryllis@yahoo.com
}

Received: September 20, 2016

Accepted: October 31, $2016 \quad$ Online Published: December 7, 2016

doi:10.5539/jedp.v7n1p42

URL: http://dx.doi.org/10.5539/jedp.v7n1p42

\begin{abstract}
The present study examines the development of basic attentional functions (sustained attention, selective attention, flexibility and divided attention) in 172 elementary school children (6-12 years) by using representative subtests from both a computerized (KITAP) and a traditional battery (TEA-Ch). The results indicate significant age-related improvements between 8 and 10 years in all the attentional functions irrespective of the assessment tool. Specifically, the results from both instruments converge regarding the developmental rates for divided attention, sustained attention and flexibility. However, performance scores in KITAP suggest a developmental plateau after the age of 10 years, whereas TEA-Ch subtests seem to be more sensitive to uncovering attention performance differences beyond the age of 10 .
\end{abstract}

Keywords: attention development, school-age, computerized assessment, traditional assessment

\section{Introduction}

The development of attention in typically developing school age population has recently attracted growing research interest, given the strong associations between attention, academic achievement and self-regulation of emotion and behavior (Posner \& Rothbart, 2005). Child literature is still limited compared to the relevant adult regarding the assessment of distinct attention functions or processes. According to most contemporary neuropsychological models, attention is a multidimensional system consisting of various functions, processes or components (Cohen, 1993; Mirsky, Anthony, Duncan, Ahearn, \& Kellam, 1991). Furthermore, developmental studies have provided convincing evidence that the development of attention is a multistage process, in which several components follow different timelines (Anderson, 2008). These timelines start from infancy and continue until adolescence, with most remarkable and rapid changes occurring during childhood (see Klenberg, Korkman, \& Lahti-Nuuttila, 2001).

Early developmental studies have tried to confirm the adult subdivision of the attention system in children and to provide developmental rates for each component with the use of tasks primarily designed for adults (Kelly, 2000; Rebok, Smith, Pascualvaca, Mirksy, Anthony, \& Kellam, 1997). Recently, more child-friendly tasks have been developed and implemented in an attempt to overcome the lack of motivation evidenced in testing children with adult material (e.g., CogState by Betts, McKay, Maruff, \& Anderson, 2006; TEA-Ch by Manly, Robertson, \& Nimmo-Smith, 1999; KI-TAP by Zimmermann, Gondan, \& Fimm, 2002). Only recently there have been empirical attempts to examine the development of children's attention as a multidimensional construct with the use of test batteries that assess various attention functions. Assessment issues have been raised regarding the ecological validity of the attention batteries as well as their sensitivity in taping the developmental trends in school age population. Specifically, traditional paper-and-pencil assessment tools have the advantage to mimic real world situations (Chevignard, Soo, Galvin, \& Catroppa, 2012), but administration and performance recording heavily depend on the examiner's experience. Computerized evaluation, instead, offers time accuracy, reliable, randomized presentation (Schatz \& Browndyke, 2002) and can override the immature verbal abilities of younger children through its button pressing requirements. Convenient though these may be, they render computer-administered batteries artificial. Thus, although these laboratory-based measurements of attention have been proved useful, considering young children's limited verbal abilities (Betts et al., 2006; Sobeh \& Spijkers, 2013), they have been criticized regarding their ecological validity (Huges \& Graham, 2002). 
The present study aimed at providing developmental data for various attention functions (sustained, selective, divided, and shift of attention) from school age population by using two different types of assessment: traditional and computerized. To our knowledge, no empirical study has provided developmental data for different attention functions that are simultaneously assessed by paper-pencil and computerized tasks. The question raised is whether there is a systematic pattern in the development of different attention functions in typically developing school children, irrespective of the assessment tool, or one assessment tool is more sensitive to capturing differences in attention performance compared to the other. Such evidence would, first, broaden our understanding regarding the consistency of developmental timelines of various attention functions in a period of rapid changes in the development of attention system (see Vakil, Blachstein, Sheinman, \& Greenstein, 2009) and, second, inform us about the sensitivity of different assessment tools in capturing age-related differences in attention.

\subsection{Developmental Evidence from Typically Developing School Children}

As mentioned above, attention represents a complex system that can be separated into several specific functions. Even though various terms have been used in the literature to describe attention functions, well-known theoretical models generally support similar representations. Namely, the two widely endorsed neuropsychological models of attention (networks of attention, Posner \& Peterson, 1990; aspects of attention, van Zomeren \& Brouwer, 1994) support the subdivision of the attention system into three basic functions or components: (a) the intensity of attention component, which refers to the ability to maintain a general level of arousal and include functions like alertness, vigilance and sustained attention, (b) the visual-spatial orientation component, which is assumed to be responsible for selective function as presented in visual scanning tasks, and (c) the executive component comprising of more complex and effort-demanding functions, such as the inhibitory control (withholding task-inappropriate responses), the ability to shift the attentional focus between two tasks (flexibility), and the ability to attend both of them at once (divided attention).

Early developmental studies using adult testing material, have reported a rapid improvement in three functions (selection, maintenance and shift) mostly at ages 8 to 10, rather than 10 to 13 (Rebok et al., 1997). A few years later, Kelly (2000) based on a sample of 100 British children aged from 7 to 13 years old found a gradual progress in processing speed emerging from 7 to 13 years. The ability to sustain attention showed a less significant improvement between 9 to 13 years, and shift of attention had a similar developmental pattern for ages 7-8 years and 9-11 years, while a reduction in the progressing rate was observed at ages 12-13. These findings provide further support to previous evidence (Rebok et al., 1997) that rapid changes in attention functions occur at school-age, specifically between years 8 and 10 . Concurrent developmental studies with child-friendly material are based mainly on computer-assisted assessment, which seems to be promising in tapping the early emergence of attention functions in school-age and even pre-school children. In Betts and colleagues' (2006) study regarding the development of sustained attention in a sample of 57 typically developing children aged between 5 to 12 years, participants completed a game-like computer-administered battery (CogState) with varying memory load. Results indicated a significant progress in children's ability to sustain attention over extended periods of time until the age of 10 years. After that age performance remained rather stable with only minor improvement. Provided with appropriately designed test material, even children below the age of 6 years can demonstrate the ability to switch attention between two or more task sets with different rules (Dibbets \& Jolles, 2006). Different cognitive aspects of visual selective attention (visual perception, visual scanning, speed of reaction time) continue to develop until the last years of elementary school (Wassenberg, Hendriksen, Hurks, Feron, Keulers, Vles, \& Jolles, 2008). Finally, flexibility and divided attention, despite their progress throughout school-age, do not seem to stabilize before the age of 12 to 16 years (Zimmermann \& Fimm, 2002).

Recently, in an attempt to evaluate the development of attention as a multidimensional system, attention batteries have been used, paper-pencil or computerized, which assess the basic functions of attention, according to the theoretical models mentioned above. Such batteries are the Test of Attentional Performance for Children-KITAP (Zimmermann et al., 2002), a computerized battery, and the Test of Everyday Attention for Children-TEA-Ch (Manly et al., 1999), a more traditional paper-pencil neuropsychological tool. Both, KITAP and TEA-Ch, have been used primarily in neuropsychological research as comprehensive attention batteries for the identification of ADHD (i.e., Heaton et al., 2001; Kaufmann et al., 2010).

Research with KITAP in typically developing school populations is limited to only two developmental cross-cultural studies (Sobeh \& Spijkers, 2012, 2013) and one study relating attentional performance to scholastic achievement (Trautmann \& Zepf, 2012). In both developmental studies KITAP has been proved to be an age sensitive instrument in primary school children (6-12 years). The findings suggest that less complex functions like 
alertness, sustained and selective attention show early emergence in the course of development and stabilize around the age of 10. Components of executive functions (flexibility, divided attention, inhibitory control) show improvement beyond childhood, and continue until early adolescence.

In the case of TEA-Ch, typically developing population has been used only in psychometric (Chang et al., 2008; Malegiannaki, Metallidou, \& Kiosseoglou, 2014) and normative studies (Manly et al., 2001). TEA-Ch was found to be an age sensitive assessment tool for attention in different school age populations, i.e., Australian, Chinese and Greek). More specifically, Manly et al. (2001) administered TEA-Ch to an Australian sample of 293 healthy children between the ages of 6 and 16, which were divided into 6 age bands. Age significantly affected performance on each TEA-Ch subtest as predicted, although the difference in performance decreased as children matured in age. No significant impact of gender was found (see also Malegiannaki et al., 2014). Similar performance patterns were detected more recently with 158 younger Chinese participants (starting from the age of 3 years until 16 years) (Chan et al., 2008). In all three studies, most attention functions were found to improve from childhood to adolescence. Even though there are distinct developmental timetables for each attention function, it seems that the most outlined and important changes occur between 8-11 years, followed by a developmental plateau after the age of 12 (see Vakil et al., 2009).

Given that between 8-11 years there seems to be a period where significant changes in attention skills occur, the aim of the present study was to provide evidence for the development of several functions of attention during primary school. Sustained, selective, divided, and shift of attention tasks (both traditional and computerized) were used, in order to include the three basic functions of attention indicated by the theoretical models (i.e., the intensity of attention, the selectivity and the flexibility/shift of attention). The computerized tasks were selected from the KITAP battery and the traditional paper-pencil tasks from the battery of TEA-Ch. Based on previous evidence, our hypothesis was that rapid changes in attention functions would occur between 8 and 10 years.

\section{Method}

\subsection{Participants}

The sample consisted of 172 children aged from 6 to 12 years, which formed three age groups $(6-8 ; 8-10 ; 10-12$ years). Both genders were represented equally in the sample (see Table 1). Children were recruited from 10 different public primary schools from the Prefecture of Thessaloniki, in North Greece, in order to collect data from different educational environments and socioeconomic regions (West, East and Center areas of Municipality of Thessaloniki, provinces, and villages). The participants come from different educational backgrounds, based on their parents' mean years of education. The present study was approved by the Greek Ministry of Education and Lifelong Learning and all parents signed informed consent. Only children with written consent of their parents were included in the study. Participants whose parents' reported neurological abnormalities or head injury, developmental disabilities, psychiatric disorders, sensory deficiency, referrals for attention, learning difficulties or needs for special education were excluded from the present study.

Table 1. Sample description

\begin{tabular}{lcccc}
\hline $\begin{array}{l}\text { Gender } \\
(N)\end{array}$ & \multicolumn{2}{c}{$\begin{array}{c}\text { Boys } \\
84\end{array}$} & $\begin{array}{l}\text { Girls } \\
88\end{array}$ & $\begin{array}{l}\text { Total } \\
172\end{array}$ \\
\hline Age Groups & $\begin{array}{c}6-8 \text { years } \\
N=47\end{array}$ & $\begin{array}{c}8-10 \text { years } \\
N=60\end{array}$ & $\begin{array}{c}10-12 \text { years } \\
N=65\end{array}$ & \\
& $7.14(0.46)$ & $8.93(0.57)$ & $10.97(0.48)$ & $9.21(1.62)$ \\
mean age in years (SD) & $13.55(3.74)$ & $13.53(2.77)$ & $12.85(3.14)$ & $13.28(3.19)$ \\
parents' mean years of education & $11.43(3.76)$ & $10.77(2.84)$ & $10.60(3.44)$ & $10.88(3.34)$ \\
\hline
\end{tabular}

\subsection{Measurements}

Four tasks from KI-TAP and four tasks from TEA-Ch were used to assess children's attentional ability respectively. Additionally, the WISC-III Vocabulary test served as a measurement of Verbal Intelligence. 


\subsubsection{Test of Attentional Performance for Children-KITAP}

KITAP is a non-verbal computerized battery for the assessment of school-age children regarding the following attentional domains: Alertness, Distractibility, Divided Attention, Flexibility, GoNogo, Sustained attention, Vigilance and Visual Scanning. Performances on these tasks can be interpreted by the following parameters: (a) reaction time in medians, (b) standard deviation of reaction times, (c) errors (incorrect responses to critical stimuli), and (d) omissions (missed responses to critical stimuli). Administration, scoring and interpretation guidelines for KITAP are available elsewhere (see Zimmermann, Gondan, \& Fimm, 2002). For the purposes of the present study four tasks corresponding to major attentional domains were chosen:

1) Sustained Attention (The Ghosts' Ball): Maintenance of attention for an extended period of time in which ghosts of different colors appeared consecutively at different windows of a castle ruin. Children were asked to press the key as quickly as possible as soon as they detected two ghosts with the same color appearing consecutively (10min, 300 trials, with 50 critical trials).

2) Selective Attention/Distractibility (The Sad and the Happy Ghost): Selective visual attention task with distracting stimuli. Children are required to ignore the distractor stimuli (other cartoons) presented in the periphery of the visual field, while trying to distinguish the critical stimuli (sad ghost) from the non-critical ones (cheerful ghost) by pressing the key-button as quickly as possible (4 min, 80 trials).

3) Flexibility (The Dragon's House): Alternating successive attention between two dragons of different color (green and blue) that appeared on the left and right side of a center gate. In cases of false response, an acoustic feedback was provided to the child and in the subsequent trial the correct stimulus was shown (1.5min, 50 trials).

4) Divided Attention (Owls): A visual-acoustic dual-task in which the child was required to simultaneously respond to critical visual stimuli (every time the visually presented owl closed its eyes) or acoustic stimuli (every time two successive high or low presented hoots appeared) by pressing the key (4.5min, 297 trials).

\subsubsection{Test of Everyday Attention for Children-TEA-Ch}

TEA-Ch was developed for the functional assessment of attentional components in children aged from 6 to 16 years. A detailed description of the nine subtests and their test items is available elsewhere (Manly et al., 1999; 2001). Its construct validity and appropriateness for the use with Greek-speaking school population has been established (Malegiannaki et al., 2014). In the present study the following four tasks were selected as representative measurements of the attentional functions:

1) Sustained Attention (Code Transmission): Children were listening to long (10 min), monotonous series of digits from 1 to 9 randomly played on an audio tape. Every time they noticed two "5s" in a row, they had to announce the digit that was presented before. The score was the number of targets correctly announced.

2) Selective Attention (Sky Search): Children are required to identify the presence of randomly distributed target stimuli (pairs of spaceships) among distractor pairs of spaceships on a colorful A3 sheet and circle them as quickly and accurate as possible. The second part is a non-distractor motor control condition. Total score on this task is calculated by subtracting the motor control time-per-target from the sky search time-per-target.

3) Flexibility (Creature Counting): This subtest required children to repeatedly alternate between counting upward and downward (i.e., from 1 to 12 and vice versa) in response to visual stimuli. Score was calculated from the sum of correct trials divided by total time taken to complete the trials.

4) Divided Attention (Sky Search DT): A dual attention task in which the visual selective attention subtest Sky Search is combined to an acoustical task of silently counting tones (the Score! subtest) and announcing them at the end of each trial. Final score on this subtest reflects the double task decrement on performance.

\subsubsection{Verbal Intelligence}

The vocabulary subtest of the standardized WISC-III Greek Version (Georgas, Paraskevopoulos, Bezevengis, \& Giannitsas, 1997) was administered as the most representative measure of verbal intelligence. The Vocabulary subtest was chosen as an alternative not time consuming option, due to its high correlation with the general IQ score (Jensen, 2001). 


\subsection{Procedure}

The present study was approved by the Greek Ministry of Education and Lifelong Learning. Authors also obtained permission from Psytest $\subset$ and Pearson Psychological Assessments $($ ) for both the translation into Greek and administration for research purposes of KITAP and TEA-Ch, respectively. The current study was part of a larger research in which all participants were assessed with full administration of the two batteries, in two different sessions each of one hour duration.

\section{Results}

To explore developmental differences on attentional performance on KITAP and TEA-Ch subtests, two different one-way analyses of covariance (ANCOVAs) were used with the three age groups (6-8 years, 8-10 years, 10-12 years) serving as independent variables. Scaled Vocabulary score (WISC-III), and mean years of education of both parents were used as covariates. Dependent variables were the test scores of the subtests of TEA-Ch (accuracy or time measures) and KITAP parameters (median of reaction time, RT; standard deviation of reaction time, SD; errors of omission, OM; false alarm errors, ER) in each analysis, respectively. The strength between age and attentional performance was estimated by using partial eta squares $\left(\eta^{2}\right)$ effect sizes. The score of errors in the Sustained attention subtest of KITAP indicated non-normal distribution (skewness $=2.05$ and kurtosis $=5.16$ ) which was corrected by using the log10-transformation, in order to meet normality requirements according to relevant literature criteria (corrected values of skewness $=.03$ and kurtosis $=-.41)($ De Carlo, 1997; West, Finch, \& Curran, 1995). Bonferroni significant difference test was used in the post hoc multiple comparisons of mean performance between age groups.

\subsection{Age Differences}

After controlling for Verbal Intelligence (Vocabulary WISC-III) and mean parents' educational level, results of ANCOVAs showed a main effect of age on children's attentional performance in KITAP and TEA-Ch subtests. As children mature in age, attentional performance improves significantly.

\subsubsection{KITAP}

More specifically, in KITAP age had a significant effect in all subtests' parameters of performance, except for the omission errors in the distractibility task (see Table 2). Flexibility and sustained attention subtests showed a stronger age effect with higher effect sizes as compared to the other two subtests. Divided Attention was also affected by age in most parameters. In general, the above results suggest that selective attention emerges early at the developmental course and shows a gradual but minor improvement as compared to the more complex executive (i.e., divided and flexibility) and intense demanding (i.e., sustained) attentional aspects. Moreover, effect sizes differed regarding subtest parameters. Generally, speed of processing (RTs and SDs) indices were more age-sensitive than indices of accuracy (omissions and errors).

Considering post hoc comparisons of the three age groups (Table 2), most significant differences were found between the younger and the older age group (1-3) in all parameters except for the stability of performance in the Divided attention task (SD). Differences between Group 1 and 2 were significant in all subtests, except for errors and RT in the less age-affected Distractibility subtest and omissions in the Sustained attention task, which showed no difference. Age differences between the older age bands (2-3) were found in most of the cases non significant or very small at best, indicating a potential developmental plateau after the age of 10 years for all functions, apart from the sustained attention. Group 2 differed from Group 3 regarding ER Distractibility and RT in Flexibility as well.

Table 2. Performance of the three age groups on KITAP subtests, results of ANCOVA and post hoc comparisons

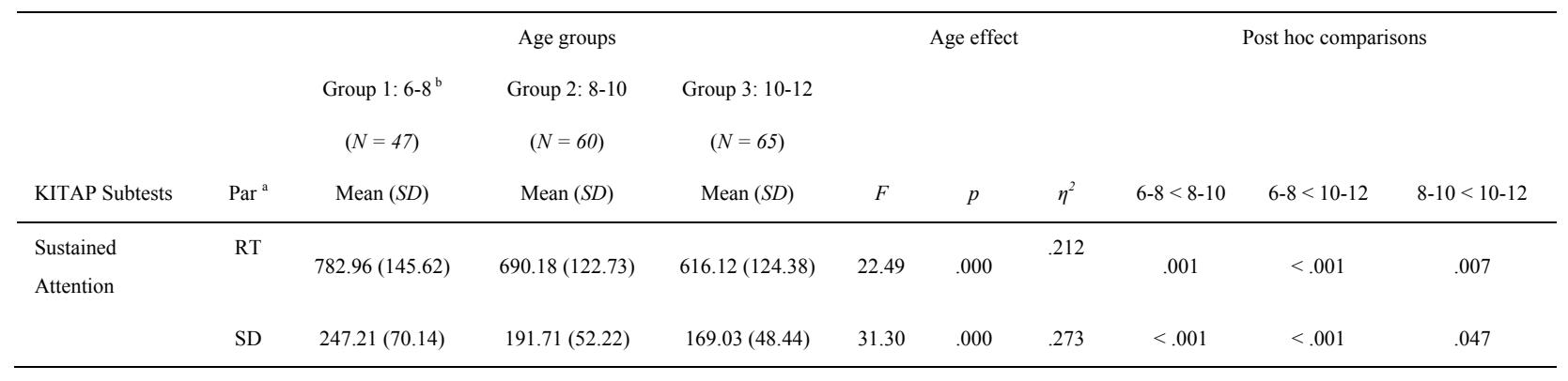




\begin{tabular}{|c|c|c|c|c|c|c|c|c|c|c|}
\hline & $\mathrm{OM}$ & $11.28(7.55)$ & $9.10(5.66)$ & $6.29(5.98)$ & 11.38 & .000 & .120 & $n s$ & $<.001$ & .022 \\
\hline & ER & $15.11(14.66)$ & $10.35(9.93)$ & $7.03(8.61)$ & 9.20 & .000 & .099 & .039 & $<.001$ & $n s$ \\
\hline $\begin{array}{l}\text { Selective } \\
\text { Attention/ }\end{array}$ & RT & $504.62(158.07)$ & 459.25 (131.99) & $440.16(125.05)$ & 3.11 & .047 & .036 & $n s$ & .047 & $n s$ \\
\hline \multirow[t]{3}{*}{ Distractibility } & $\mathrm{SD}$ & $359.02(242.81)$ & $231.75(164.56)$ & $249.20(233.79)$ & 5.98 & .003 & .067 & .005 & .013 & $n s$ \\
\hline & $\mathrm{OM}$ & $4.09(3.61)$ & $3.08(2.84)$ & $3.02(3.02)$ & 1.86 & $n s$ & - & - & - & - \\
\hline & ER & $20.26(7.93)$ & $16.80(8.36)$ & $13.12(8.26)$ & 11.57 & .000 & .122 & $n s$ & $<.001$ & .029 \\
\hline \multirow[t]{3}{*}{ Flexibility } & RT & $1310.02(405.02)$ & $1035.57(279.23)$ & $855.97(210.60)$ & 31.43 & .000 & .273 & $<.001$ & $<.001$ & .004 \\
\hline & $\mathrm{SD}$ & $525.06(238.34)$ & $348.65(137.53)$ & $281.89(129.22)$ & 31.51 & .000 & .274 & $<.001$ & $<.001$ & $n s$ \\
\hline & ER & $5.47(3.64)$ & $2.97(2.73)$ & $2.23(1.91)$ & 23.18 & .000 & .217 & $<.001$ & $<.001$ & $n s$ \\
\hline \multirow[t]{4}{*}{ Divided Attention } & $\mathrm{RT}$ & 793.31 (93.24) & $699.88(113.56)$ & $674.85(79.31)$ & 21.63 & .000 & .206 & $<.001$ & $<.001$ & $n s$ \\
\hline & $\mathrm{SD}$ & $281.97(85.40)$ & $234.80(64.09)$ & $253.50(78.27)$ & 5.26 & .006 & .059 & .004 & $n s$ & $n s$ \\
\hline & $\mathrm{OM}$ & $5.91(5.33)$ & $3.80(3.66)$ & $3.31(4.00)$ & 5.83 & .004 & .065 & .030 & .004 & $n s$ \\
\hline & ER & $15.70(17.05)$ & $8.58(10.48)$ & $8.08(10.67)$ & 7.31 & .001 & .081 & .005 & .001 & $n s$ \\
\hline
\end{tabular}

Notes. ${ }^{\text {a }}$ Par. $=$ Parameter; $\mathrm{RT}=$ median of the reaction time; $\mathrm{SD}=$ standard deviation of reaction times; $\mathrm{OM}=$ omissions; $\mathrm{ER}=$ false alarm errors.

${ }^{\mathrm{b}}$ Represents the upper cutoff of each band (e.g., only children under 8 years would appear in group "6-8”); $n s=$ not significant.

\subsubsection{TEA-Ch}

Performance on TEA-Ch subtests was significantly affected by age. Similarly to KITAP, older children tended to score higher when compared to younger age groups as expected. Unlike the case with KITAP, post hoc comparisons of TEA-Ch showed an ongoing development of attentional performance until 12 years (see Table 3). The only non significant differences were observed between Group 2 and Group 3 performance on Sky Search DT subtest.

Table 3. Performance of the three age groups on TEA-Ch Subtests, Results of ANCOVA and post hoc comparisons

\begin{tabular}{|c|c|c|c|c|c|c|c|c|c|}
\hline \multirow[b]{3}{*}{ TEA-Ch Subtests } & \multicolumn{3}{|c|}{ Age groups } & \multicolumn{3}{|c|}{ Age } & \multicolumn{3}{|c|}{ Post hoc comparisons } \\
\hline & $\begin{array}{l}\text { Group 1: 6-8 } \\
\qquad(N=47)\end{array}$ & $\begin{array}{c}\text { Group 2: 8-10 } \\
\qquad(N=60)\end{array}$ & $\begin{array}{l}\text { Group 3: } 10-12 \\
\qquad(N=65)\end{array}$ & & & & & & \\
\hline & Mean $(S D)$ & Mean $(S D)$ & Mean $(S D)$ & $F$ & $p$ & $\eta^{2}$ & $6-8<8-10$ & $6-8<10-12$ & $8-10<10-12$ \\
\hline \multicolumn{10}{|l|}{ Sustained } \\
\hline \multicolumn{10}{|l|}{ Attention } \\
\hline (accuracy) & $30.28(6.35)$ & 34.58 (3.59) & $36.72(3.23)$ & 31.38 & .000 & .273 & $p<.001$ & $p<.001$ & $p<.05$ \\
\hline \multicolumn{10}{|l|}{ Selective } \\
\hline \multicolumn{10}{|l|}{ Attention } \\
\hline (time-per-target) & $6.87(2.17)$ & $5.21(1.59)$ & $4.21(1.05)$ & 40.73 & .000 & .328 & $p<.001$ & $p<.001$ & $p<.005$ \\
\hline \multicolumn{10}{|l|}{ Flexibility } \\
\hline (time-per-shift) & $6.74(2.87)$ & $5.18(1.73)$ & $3.90(0.93)$ & 35.63 & .000 & .299 & $p<.001$ & $p<.001$ & $p<.001$ \\
\hline \multicolumn{10}{|l|}{ Divided } \\
\hline \multicolumn{10}{|l|}{ Attention } \\
\hline (decrement) & $10.91(11.29)$ & $4.71(8.82)$ & $2.36(3.13)$ & 16.95 & .000 & .169 & $p<.001$ & $p<.001$ & $n s$ \\
\hline
\end{tabular}

Notes. " Represents the upper cutoff of each band (e.g., only children under 8 years would appear in group "6-8"); $n s=$ not significant. 


\section{Discussion}

The present study aimed at exploring the development of attention functions in school age children by using both computerized and paper-pencil assessment tools. One of the strengths of the study is that it utilized child-friendly behavioral tasks in a sample of typically developing children. The present results indicated that both assessment tools are age-sensitive in assessing the basic attention functions in school age years.

Regarding selective attention, the results from the KITAP subtests indicated a slow but gradual development occurring primarily between 6-10 years, where the plateau is formed. Only omissions of the target stimulus showed no significant age improvement (see also Wassenberg et al., 2008). Contrary to that, the time-based measurement of TEA-Ch selective attention task, suggested an improvement beyond that age point. More light on this controversy could be shed by Wassenberg's et al. (2008) study in which the $\mathrm{d} 2$ test of selective attention was administered to 451 Dutch elementary school children. Their outcomes support a differential developmental pattern of certain aspects of selective attention as obtained by the $\mathrm{d} 2$ task parameters. More specifically, the level of inattention as indicated by number of omissions remained stable across all grades. The impulsivity aspect, as measured by false alarm errors, had an age-related improvement until the fourth grade. Processing speed of the d2 test continued developing until the sixth school grade. To sum up, in the present study stability of inattention performance was evidenced using the KITAP selective attention subtest, whereas the continuing progress of the speed parameter of selective attention was revealed by the respective TEA-Ch subtest. According to Wassenberg et al. (2008) this is explained by the early presence of basic and non-complex elements of executive functions, which will eventually develop into a systematical goal-directed behavior during childhood and until adolescence. Discrepancies between locating the plateau on $4^{\text {th }}$ or $6^{\text {th }}$ school grade are assumed to possibly reflect differences regarding assessment methodology, paper-pencil as the d2 test, or computerized (e.g., Klimkeit, Mattingley, Sheppard, Farrow, \& Bradshaw, 2004). At this point, the current study extends knowledge regarding the comparison of these two types of assessment. Thus, in our study the RT score of KITAP distractibility subtest stabilized around the age of 10 , while with TEA-Ch time score in selective attention task suggested the improvement of reaction time beyond that age point.

No discrepancy was observed between the two assessment tools regarding the sustained attention. Detecting age-related changes of sustained attention beyond the stabilization point of 10 years is a step forward in the assessment with computerized test material. For example, Betts et al. (2006) using a novel program (CogState) found that sustained attention improved until 10 years followed by a plateau. Researchers claimed that the output of computer-administered batteries offer the opportunity of calculating more complex parameters, such as the variability (for example $S D$ ), which along with RT, are assumed to be more indicative of the assessment of sustained attention. In the TEA-Ch battery, the two available pure sustained attention tasks (Score! and Code Transmission, which were included in the present study) provide only accuracy scores. Such indices (errors of omission or false alarm and correct answers) may be less representative of young children's sustained attention performance since variability in response is a commonly observed phenomenon. In this framework, ceiling or floor effects (Manly et al., 2001) can easily occur in the assessment, and should be carefully interpreted when examining developmental changes (Betts et al., 2006).

In the case of divided attention the developmental plateau after the age of 10 was evidenced by both assessment tools. The results converge with Sobeh and Spijker's (2013) findings with a Syrian and German sample of 562 children from 5 to 12 years. Given that divided attention represents an effortful executive control aspect, it would be a mistake to conclude that divided attention does not develop at all after the age of 10. As already stated by Klenberg et al. (2001) "Some developmental changes could occur before and some after the developmental peak".

Finally, as regards the flexibility or shifting attention component, the present results from both measures showed that the reaction time continues to decrease until the age of 12 years, which suggests a gradual development of this aspect of attention. Similar developmental trends for the switching ability have been reported in previous research (see Zimmermann \& Fimm, 2002) using the time-based estimates (RTs) of the Flexibility subtest. In fact, this subtest has been indicated as the most age-sensitive attention task of the overall KITAP battery (correlating $r=-.66$ to age) and, despite early emergence its development was found to stabilize after the age of 14 or 15 years.

Given the limitation of the cross-sectional design of the present study, the results indicate that, irrespective of the assessment tool, there is a significant development in basic attention functions through the primary school years, especially between 8 and 10 years. Performance scores in KITAP suggest a developmental plateau in most of the parameters after the age of 10 years, except for the sustained attention. TEA-Ch subtests seem to be more sensitive in uncovering attention performance differences beyond the age of 10 years, with the exception of the divided 
attention task. This is not unexpected, though, since TEA-Ch was initially developed to assess children's attention up to the age of 16 years, while KITAP up to the age of 10 years (even though it has also been administered to adolescents). Task-specific characteristics in traditional assessment, such as the verbal modality may raise task complexity and confound attention assessment with other cognitive factors, i.e., language and working memory. Thus, some TEA-Ch subtests characteristics (e.g., the divided attention subtest) might be suggested as an examination tool suitable for older children. Taking into consideration that academic underachievement has been consistently associated with attention problems and that the attention network is considered very important for self-regulation of emotion and behavior (Rothbart \& Rueda, 2005), the present results indicate that is crucial to provide researchers and educators with child-friendly, age-sensitive behavioral assessment tools. Further, they indicate the importance of finding ways to foster attention skills, especially in periods where significant changes in attention skills occur.

\section{Acknowledgements}

This study was supported by the Alexander S. Onassis Public Benefit Foundation for the doctoral research of Amaryllis-Chryssi Malegiannaki.

\section{References}

Anderson, P. (2008). Towards a developmental theoretical framework for executive function. In V. Anderson, R. Jacobs, \& P. Anderson (Eds.), Executive Functions and the Frontal Lobes: A Lifespan Perspective (pp. 3-22). New York, NY: Taylor \& Francis Group.

Betts, J., McKay, J., Maruff, P., \& Anderson, V. (2006). The development of sustained attention in children: The effect of age and task load. Child Neuropsychology, 12, 205-211. https://dx.doi.org/10.1080/09297040500488522

Brocki, K. C., \& Bohlin, G. (2004). Executive functions in children aged 6-13: A dimensional and developmental study. Developmental Neuropsychology, 26, 571-593. https://dx.doi.org/10.1207/s15326942dn2602_3

Chan, R. C. K., Wang, L., Ye, J., Leung, W. W. Y., \& Mok, M. Y. K. (2008). A psychometric study of the Test of Everyday Attention for Children in the Chinese setting. Archives of Clinical Neuropsychology, 23(4), 455-466. https://dx.doi.org/10.1016/j.acn.2008.03.007

Chevignard, M. P., Soo, C., Galvin, J., Catroppa, C., \& Eren S. (2012). Ecological assessment of cognitive functions in children with acquired brain injury: A systematic review. Brain Injury, 26(9), 1033-1057. https://dx.doi.org/10.3109/02699052.2012.666366

Cohen, R. A. (1993). The neuropsychology of attention. New York: Plenum Publishing. https://dx.doi.org/10.1007/978-1-4419-7463-1

Dibbets, P., \& Jolles, J. (2006). The Switch Task for Children: Measuring mental flexibility in young children. Cognitive Development, 21, 60-71. https://dx.doi.org/10.1016/j.cogdev.2005.09.004

Georgas, D. D., Paraskevopoulos, I., Bezevengis, I., \& Giannitsas, N. (1997). Guidelines for the Greek WISC-III. Athens: Ellinika Grammata.

Heaton, S. C., Reader, S. K., Preston, A. S., Fennall, E. B., Puyana, O. E., Gill, N., \& Johnson, J. H. (2001). The Test of Everyday Attention for Children (TEA-Ch): Patterns of performance in Children with ADHD and clinical controls. Child Neuropsychology, 7, 251-264. https://dx.doi.org/10.1076/chin.7.4.251.8736

Hughes, C., \& Graham, A. (2002). Measuring executive functions in childhood: Problems and Solutions? Child and Adolescent Mental Health, 7, 131-142. https://dx.doi.org/10.1111/1475-3588.00024

Jensen, A. R. (2001). Vocabulary and general intelligence. Behavioral and Brain Sciences, 24(6), 1109-1110. https://dx.doi.org/10.1017/S0140525X01280133

Kaufmann, L., Zieren, N., Zotter, S., Karall, D., Scholl-Bürgi, S., Haberlandt, E., \& Fimm, B. (2010). Predictive validity of attentional functions in differentiating children with and without ADHD: A componential analysis. $\begin{array}{llll}\text { Developmental Medicine } \& \text { Child Neurology, } & \text { 52(4), }\end{array}$ https://dx.doi.org/10.1111/j.1469-8749.2009.03560.x

Kelly, T. P. (2000). The clinical neuropsychology of attention in school-aged children. Child Neuropsychology, 6 , 24-36. https://dx.doi.org/10.1076/0929-7049(200003)6:1;1-B;FT024 
Klenberg, L., Korkman, M., \& Lahti-Nuuttila, P. (2001). Differential development of attention and executive functions in 3- to 12-year-old Finnish children. Developmental Neuropsychology, 20, 407-428. https://dx.doi.org/10.1207/S15326942DN2001_6

Klimkeit, E. I., Mattingley, J. B., Sheppard, D. M., Farrow, M., \& Bradshaw, J. L. (2004). Examining the development of attention and executive functions in children with a novel paradigm. Child Neuropsychology, 10, 201-211. https://dx.doi.org/10.1080/09297040409609811

Malegiannaki, A. C., Metallidou, P., \& Kiosseoglou, G. (2014). Psychometric properties of the Test of Everyday Attention for Children in Greek-speaking school children. European Journal of Developmental Psychology: Developmetrics, 12(2), 234-242. http://dx.doi.org/10.1080/17405629.2014.973842

Manly, T., Robertson, I. H., Anderson, V., \& Nimmo-Smith, I. (1999). The Test of Everyday Attention for Children: Manual. Bury St. Edmunds, UK: Thames Valley Test Company, Ltd.

Manly, T., Robertson, I. H., Anderson, V., \& Nimmo-Smith, I. (2001). The Differential assessment of children's attention: The Test of Everyday Attention for Children (TEA-Ch), normative sample and ADHD performance. Journal of Child Psychology and Psychiatry, 42(8), 1065-1081. https://dx.doi.org/10.1111/1469-7610.00806

Mirsky, A. F., Anthony, B. J., Duncan, C. C., Ahearn, M. B., \& Kellam, S. G. (1991). Analysis of the elements of attention: A neuropsychological approach. Neuropsychology Review, 2, 109-145. https://dx.doi.org/10.1007/BF01109051

Posner, M. I., \& Rothbart, M. K. (2005). Educating the Human Brain. Washington, DC: American Psychological Association.

Rebok, G. W., Smith, C. B., Pascualvaca, D. M., Mirsky, A. F., Anthony, B. J., \& Kellam, S. G. (1997). Developmental changes in attentional performance in urban children from eight to thirteen years. Child Neuropsychology, 3, 28-46. https://dx.doi.org/10.1080/09297049708401366

Robertson, I. H., Ward, A., Ridgeway, V., \& Nimmo-Smith, I. (1994). Test of Everyday Attention. Bury St. Emunds, U.K.: Thames Valley Test Company.

Rothbart, M. K., \& Rueda, M. R. (2005). The development of effortful control. In U. Mayr, \& S. W. Awh (Eds.), Developing Individuality in the Human Brain: A Festschriftt Honoring Michael I. Posner (pp. 167-188). Washington, DC: American Psychological Association. https://dx.doi.org/10.1037/11108-009

Schatz, P., \& Browndyke, J. (2002). Applications of computer-based neuropsychological assessment. The Journal of Head Trauma Rehabilitation, 17(5), 395-410. https://dx.doi.org/10.1097/00001199-200210000-00003

Sobeh, J., \& Spijkers, W. (2012). Development of attention functions in 5- to 11-year-old Arab children as measured by the German Test Battery of Attention Performance (KITAP): A pilot study from Syria. Child Neuropsychology, 18(2), 144-167. https://dx.doi.org/10.1080/09297049.2011.594426

Sobeh, J., \& Spijkers, W. (2013). Development of neuropsychological functions of attention in two cultures: A cross-cultural study of attentional performances of Syrian and German children of pre-school and school age. European Journal of Developmental Psychology, 10(3), 318-336. https://dx.doi.org/10.1080/17405629.2012.674761

Trautmann, M., \& Zepf, F. D. (2012). Attentional performance, age and scholastic achievement in healthy children. PLoS ONE, 7(3), e32279. https://dx.doi.org/10.1371/journal.pone.0032279

Vakil, E., Blachstein, H., Sheinman, M., \& Greenstein, Y. (2009). Developmental changes in attention test norms: Implications for the structure of attention. Child Neuropsychology, 15, 21-39. https://dx.doi.org/10.1080/09297040801947069

Van Zomeren, A. H., \& Brouwer, W. H. (1994). Clinical neuropsychology of attention. New York, NY: Oxford University Press.

Verstraeten, K., Vasey, M. W., Claes, L., \& Bijttebier, P. (2010). The assessment of effortful control in childhood: Questionnaires and the Test of Everyday Attention for Children compared. Personality and Individual Differences, 48(1), 59-65. https://dx.doi.org/10.1016/j.paid.2009.08.016

Wassenberg, R., Hendriksen, J. G. M., Hurks, P. P. M., Feron, F. J. M., Keulers, E. H. H., Vles, J. S. H., \& Jolles, J. (2008). Development of inattention, impulsivity, and processing speed as measured by the $\mathrm{d} 2$ test: Results 
of a large cross-sectional study in children aged 7-13. Child Neuropsychology, 14, $195-210$. https://dx.doi.org/10.1080/09297040601187940

Zimmermann, P., \& Fimm, B. (2002). A test battery for attentional performance. In M. Leclercq, \& P.

Zimmermann (Eds.), Applied neuropsychology of attention: Theory, diagnosis and rehabilitation (pp. 110-151). London, UK: Psychology Press.

Zimmermann, P., Gondan, M., \& Fimm, B. (2002). Testbatterie zur Aufmerksamkeitsprüfung für Kinder (KITAP) [Children Version Test-battery of Attention Performance (KITAP)]. Würselen, Germany: Psytest.

\section{Copyrights}

Copyright for this article is retained by the author(s), with first publication rights granted to the journal.

This is an open-access article distributed under the terms and conditions of the Creative Commons Attribution license (http://creativecommons.org/licenses/by/4.0/). 